\title{
ON THE METRIC PROJECTION ONTO PROX-REGULAR SUBSETS OF RIEMANNIAN MANIFOLDS
}

\author{
SEYEDEHSOMAYEH HOSSEINI AND MOHAMAD R. POURYAYEVALI \\ (Communicated by Sergei K. Suslov) \\ Abstract. Prox-regular subsets of Riemannian manifolds are introduced. A \\ characterization of prox-regular sets based on the hypomonotonicity of the \\ truncated limiting normal cone is obtained. Moreover, some properties of \\ metric projection mapping and distance function corresponding to the prox- \\ regular sets are presented.
}

\section{INTRODUCTION}

It is well known that for a closed convex set $S$ in a Hilbert space $\mathbb{H}$, the metric projection is Lipschitz and the distance function from $S$ is differentiable everywhere outside of $S$. It was shown in [19] that for a class of (not necessarily convex) sets, the corresponding metric projection exists and is Lipschitz in a neighborhood of each set. These sets were studied under the name of "prox-regular" sets in [17. It was proved in [18, that the prox-regularity is a necessary and sufficient condition for existence and Lipschitz continuity of metric projection. Moreover, the authors established a characterization of prox-regular sets in Hilbert spaces based on the hypomonotonicity of the truncated limiting normal cone.

Unlike a Hilbert space, a manifold in general does not have a linear structure, and therefore new techniques are needed for dealing with the concepts of metric projection and distance function from sets in manifolds. Moreover, these notions are not of local type and cannot be studied by local techniques. A number of results regarding metric projection and distance function corresponding to the convex sets in Riemannian manifolds have been obtained. In [21] the differentiability of the metric projection for a closed locally convex subset $S$ of a finite dimensional Riemannian manifold $M$ was shown. Moreover, the author proved that the distance function from $S$ near and outside of $S$ is of class $C^{1}$.

In 1981, Greene and Shiohama proved that for a closed totally convex set $S$ of a finite dimensional Riemannian manifold $M$, there exists an open set $W$ containing $S$ such that the metric projection is locally Lipschitz on $W$; see $[8$. It was shown in [9] that for a closed convex set $S$ of a finite dimensional Hadamard manifold $M$, the metric projection is single-valued and Lipschitz on $M$.

Received by the editors December 18, 2010.

2010 Mathematics Subject Classification. Primary 49J52, 58C06, 58C20.

Key words and phrases. Prox-regularity, Riemannian manifolds, Clarke subdifferential, proximal subdifferential.

The second author was partially supported by the Center of Excellence for Mathematics, University of Shahrekord, Iran. 
The concept of a monotone vector field on Riemannian manifolds which was a generalization of a monotone operator was introduced in [16. The relationship between convexity and monotonicity has been studied in numerous papers such as 22 .

Our goal is to generalize the results regarding metric projection of totally convex subsets obtained in [8] to prox-regular subsets of Riemannian manifolds. We introduce the notions of regular sets and regular functions on manifolds. Then a subclass of regular sets named prox-regular which contains convex sets is introduced. Moreover, hypomonotone mappings on convex subsets of a Riemannian manifolds are considered and by using these mappings a characterization for prox-regular sets is obtained. Other properties of prox-regular sets related to the corresponding metric projection and distance function are given. In fact, we prove that for a prox-regular subset $S$ of a Riemannian manifold $M$ there exists an open set $W$ containing $S$ such that the metric projection is a single-valued continuous mapping on $W$. Moreover, we show that if $M$ is a Hadamard manifold, then the metric projection is locally Lipschitz on $W$ and the distance function from $S$ is $C^{1+}$ on $W \backslash S$.

\section{Preliminaries}

In this paper, we use the standard notation and known results of Riemannian manifolds; see, e.g., 6, 11, 13. Throughout this paper, $M$ is a $C^{\infty}$ finite dimensional manifold endowed with a Riemannian metric $\langle., .\rangle_{x}$ on the tangent space $T_{x} M \cong \mathbb{R}^{n}$.

As usual we denote by $B(x, \delta)$ the open ball centered at $x$ with radius $\delta$ and by $\bar{N}$ the closure of the set $N$. Let $S$ be a nonempty closed subset of a Riemannian manifold $M$. We define $d_{S}: M \longrightarrow \mathbb{R}$ by

$$
d_{S}(x):=\inf \{d(x, s): s \in S\},
$$

where $d$ is the Riemannian distance on $M$.

We will make extensive use of the exponential mapping $\exp _{x}$ throughout the paper. Recall that for every $x \in M$ there exists a mapping $\exp _{x}$, defined on a neighborhood of $0_{x}$ in the tangent space $T_{x} M$, and taking values in $M$, which is a local diffeomorphism and maps straight line segments passing through $0_{x} \in T_{x} M$ onto geodesic segments in $M$ passing through $x$. Any open neighborhood $U$ of $x \in M$ that is the diffeomorphic image under $\exp _{x}$ of an open neighborhood of $0_{x}$ is called a normal neighborhood of $x$. We will also use the parallel transport of vectors along geodesics. For a given curve $\gamma: I \rightarrow M$, number $t_{0} \in I$, and a vector $V_{0} \in T_{\gamma\left(t_{0}\right)} M$, there exists a unique parallel vector field $V(t)$ along $\gamma(t)$ such that $V\left(t_{0}\right)=V_{0}$. Moreover, the mapping defined by $V_{0} \mapsto V\left(t_{1}\right)$ is a linear isometry between the tangent spaces $T_{\gamma\left(t_{0}\right)} M$ and $T_{\gamma\left(t_{1}\right)} M$, for each $t_{1} \in I$. In the case when $\gamma$ is a minimizing geodesic and $\gamma\left(t_{0}\right)=x, \gamma\left(t_{1}\right)=y$, we will denote this mapping by $L_{x y}$, and we will call it the parallel transport from $T_{x} M$ to $T_{y} M$ along the curve $\gamma$. Note that $L_{x y}$ is well defined when the minimizing geodesic which connects $x$ to $y$ is unique. In what follows, $L_{x y}$ will be used wherever it is well defined.

Recall that a subset $S$ of a Riemannian manifold $M$ is called convex if every two points $p_{1}, p_{2} \in S$ can be joined by a unique geodesic whose image belongs to $S$. Moreover, for every convex subset $U$ of a Riemannian manifold $M$ and arbitrary points $p_{1}, p_{2}$ in $U$, $\exp _{p_{i}}^{-1}$, for $i=1,2$ is well-defined on $U$ and

$$
\left\|\exp _{p_{1}}^{-1}\left(p_{2}\right)\right\|=d\left(p_{1}, p_{2}\right) .
$$


Now we present some definitions and results in nonsmooth analysis on Riemannian manifolds; for more details see 10 .

Let $M$ be a Riemannian manifold, $x \in M$ and let $f: M \rightarrow \mathbb{R}$ be a locally Lipschitz function. The Clarke subdifferential of $f$ at $x$, denoted by $\partial_{C} f(x)$, is defined as follows:

$$
\partial_{C} f(x)=\partial_{C}\left(f \circ \exp _{x}\right)\left(0_{x}\right),
$$

where $\partial_{C}\left(f \circ \exp _{x}\right)\left(0_{x}\right)$ is the Clarke subdifferential of $f \circ \exp _{x}$ at $0_{x}$ as a locally Lipschitz function defined on a subset of $T_{x} M$.

Let $S$ be a nonempty closed subset of a Riemannian manifold $M, x \in S$ and let $(\varphi, U)$ be a chart of $M$ at $x$. Then the (Clarke) tangent cone to $S$ at $x$, denoted by $T_{S}^{C}(x)$, is defined as follows:

$$
T_{S}^{C}(x):=d \varphi(x)^{-1}\left[T_{\varphi(S \cap U)}^{C}(\varphi(x))\right],
$$

where $T_{\varphi(S \cap U)}^{C}(\varphi(x))$ is a tangent cone to $\varphi(S \cap U)$ as a subset of $\mathbb{R}^{n}$ at $\varphi(x)$. Note that the definition of $T_{S}(x)$ does not depend on the choice of the chart $\varphi$ at $x$; see [15. Lemma 3.4].

Obviously, $0_{x} \in T_{S}^{C}(x)$ and $T_{S}^{C}(x)$ is closed and convex. In the case of submanifolds of $\mathbb{R}^{n}$, the tangent space and the normal space are orthogonal to each other. In an analogous manner, for a closed subset $S$ of a Riemannian manifold $M$ the normal cone to $S$ at $x$, denoted by $N_{S}^{C}(x)$, is defined as the (negative) polar of the tangent cone $T_{S}^{C}(x)$, i.e.

$$
N_{S}^{C}(x):=T_{S}^{C}(x)^{\circ}:=\left\{\xi \in T_{x} M^{*}:\langle\xi, z\rangle \leq 0 \quad \forall z \in T_{S}^{C}(x)\right\} .
$$

It follows from [5, Proposition 2.5.2] that $v \in T_{S}^{C}(x)$ if and only if for every normal neighborhood $U$ of $x$ and every sequence $\left(z_{i}\right) \subset \exp _{x}^{-1}(S \cap U)$ converging to $0_{x}$ and sequence $t_{i}$ in $(0, \infty)$ decreasing to 0 , there exists a sequence $\left(v_{i}\right) \subset T_{x} M$ converging to $v$ such that for all $i, z_{i}+t_{i} v_{i} \in \exp _{x}^{-1}(S \cap U)$.

Let $M$ be a Riemannian manifold, $x \in M$ and $f: M \rightarrow(-\infty,+\infty]$ be a lower semicontinuous function. The proximal subdifferential of $f$ at $x$, denoted by $\partial_{P} f(x)$, is defined as $\partial_{P}\left(f \circ \exp _{x}\right)\left(0_{x}\right)$.

As a consequence of the definition of $\partial_{P}\left(f \circ \exp _{x}\right)\left(0_{x}\right)$ one has that $v \in \partial_{P} f(x)$ if and only if there is $t>0$ such that

$$
f(y) \geq f(x)+\left\langle v, \exp _{x}^{-1}(y)\right\rangle-t / 2 d(x, y)^{2}
$$

for every $y$ in a neighborhood of $x$.

Suppose now that $S$ is a closed subset of a Riemannian manifold $M$ and that $x \in S$. We define the proximal normal cone to $S$ at $x$, denoted by $N_{S}^{P}(x)$, as $N_{\exp _{x}^{-1}(U \cap S)}^{P}\left(0_{x}\right)$, where $U$ is any normal neighborhood of $x$.

The following lemma is an easy consequence of the definition of $N_{S}^{P}(x)$.

Lemma 2.1. Let $S$ be a closed subset of a Riemannian manifold $M$ and let $x \in S$. Then $v \in N_{S}^{P}(x)$ if and only if there is $\rho>0$ such that

$$
\left\langle v, \exp _{x}^{-1}(y)\right\rangle \leq \rho / 2 d(y, x)^{2},
$$

for every $y$ in a neighborhood of $x$.

Remark 1. It is easy to verify that $N_{S}^{P}(x)=\partial_{P} \delta_{S}(x)$; here $\delta_{S}$ is the indicator function of $S$ defined by $\delta_{S}(x)=0$ if $x \in S$ and $\delta_{S}(x)=\infty$ if $x \notin S$. 
Let us define the limiting subdifferential of a lower semicontinuous function $f$ at $x$, denoted by $\partial_{L} f(x)$, and the limiting normal cone to a closed subset $S$ of a Riemannian manifold $M$ at $x \in S$, denoted by $N_{S}^{L}(x)$, as follows:

$$
\begin{aligned}
\partial_{L} f(x) & =\left\{\lim _{i \rightarrow \infty} v_{i}: v_{i} \in \partial_{P} f\left(x_{i}\right), x_{i} \underset{\rightarrow}{f} x\right\}, \\
N_{S}^{L}(x) & =\left\{\lim _{i \rightarrow \infty} v_{i}: v_{i} \in N_{S}^{P}\left(x_{i}\right), x_{i} \underset{\rightarrow}{S} x\right\},
\end{aligned}
$$

where $x_{i} \underset{f}{f} x$ means that $x_{i}$ and $f\left(x_{i}\right)$ are respectively convergent to $x$ and $f(x)$ and $x_{i} \underline{S} x$ denotes the convergent sequence $\left\{x_{i}\right\} \subset S$ to $x$.

The following lemma states the connections between Clarke and proximal and limiting subdifferentials. Also, it shows the relationships between normal cones.

Lemma 2.2. Let $M$ be a Riemannian manifold.

(a) If $f$ is Lipschitz near $x$, then

$$
\partial_{C} f(x)=\overline{\mathrm{co}}\left\{\lim _{i \rightarrow \infty} \xi_{i}: \xi_{i} \in \partial_{P} f\left(x_{i}\right), x_{i} \rightarrow x\right\} .
$$

(b) If $S$ is a closed subset of $M$ containing $x$, then

$$
N_{S}^{C}(x)=\overline{\mathrm{co}}\left\{\lim _{i \rightarrow \infty} \xi_{i}: \xi_{i} \in N_{S}^{P}\left(x_{i}\right), x_{i} \rightarrow x\right\},
$$

where co signifies convex hull.

We conclude this section with the definition of the Bouligand (or contingent) tangent cone to a closed subset $S$ of a Riemannian manifold $M$ at a point $x \in S$, which is a generalization of the definition of the contingent cone to a closed subset of a Banach space; see [5, p. 90]. Let $S$ be a closed subset of a Riemannian manifold $M$ and $x \in S$. The Bouligand (or contingent) tangent cone to $S$ at $x$, denoted by $T_{S}^{B}(x)$, is defined as follows:

$$
T_{S}^{B}(x):=\left\{\lim _{i \rightarrow \infty} \frac{z_{i}}{t_{i}}: z_{i} \rightarrow 0_{x} \text { and } t_{i} \downarrow 0, z_{i} \in \exp _{x}^{-1}(S \cap U)\right\},
$$

where $U$ is a normal neighborhood of $x$.

\section{MAin Results}

3.1. Regular sets and functions on Riemannian manifolds. In this subsection, we study regular sets and functions on manifolds. Moreover, prox-regular sets and primal lower nice functions on Riemannian manifolds are defined, and some related results to these concepts are proved.

Definition 3.1. A function $f: M \longrightarrow \mathbb{R}$ defined on a Riemannian manifold $M$ is said to be proximally subdifferentially regular, or regular for short, at $x \in M$ if $f$ is Lipschitz near $x$, and

$$
\partial_{L} f(x)=\partial_{C} f(x)=\partial_{P} f(x) .
$$

Definition 3.2. A closed subset $S$ of a Riemannian manifold $M$ is said to be tangentially regular, or regular for short, at $x \in S$ if

$$
T_{S}^{C}(x)=T_{S}^{B}(x) .
$$

It is worth pointing out that every convex set is a regular set. In order to illustrate nonconvex regular sets, nonconvex prox-regular sets in Riemannian manifolds are introduced. For the definitions of prox-regular sets and primal lower nice functions on Riemannian manifolds, we need to use a convex neighborhood around each point. 
The existence of this convex neighborhood is ensured by Whitehead's theorem; see [11.

Definition 3.3. A closed subset $S$ of a Riemannian manifold $M$ is said to be proxregular at $\bar{x} \in S$ if there exist $\varepsilon>0$ and $\rho>0$ such that $B(\bar{x}, \varepsilon)$ is convex and for every $x \in S \cap B(\bar{x}, \varepsilon)$ and $v \in N_{S}^{L}(x)$ with $\|v\|<\varepsilon$,

$$
\left\langle v, \exp _{x}^{-1}\left(x^{\prime}\right)\right\rangle \leq \rho / 2 d\left(x^{\prime}, x\right)^{2} \text {, for every } x^{\prime} \in S \cap B(\bar{x}, \varepsilon) .
$$

It is easy to verify that if $S$ is prox-regular at $\bar{x}$, then $N_{S}^{P}(\bar{x})$ is closed.

A closed subset $S$ of a Riemannian manifold $M$ is said to be prox-regular if it is prox-regular at each point of $S$. It is worthwhile to mention that convex sets are prox-regular.

Definition 3.4. Let $M$ be a Riemannian manifold. A lower semicontinuous function $f: M \rightarrow(-\infty,+\infty]$ is said to be primal-lower-nice (p.l.n.) at $\bar{x} \in \operatorname{dom}(f)$ if there exist $t_{0}>0, b>0$ and $\varepsilon>0$ such that $B(\bar{x}, \varepsilon)$ is convex and

$$
f\left(x^{\prime}\right) \geq f(x)+\left\langle\exp _{x}^{-1}\left(x^{\prime}\right), v\right\rangle-t / 2 d\left(x^{\prime}, x\right)^{2}
$$

provided that $t>t_{0},\|v\|<b t, v \in \partial_{P} f(x), d\left(x^{\prime}, \bar{x}\right)<\varepsilon, d(x, \bar{x})<\varepsilon$.

Note that $\operatorname{dom}(f)$ denotes the effective domain of $f$.

One can prove that if $f: M \rightarrow(-\infty,+\infty]$ is p.l.n. at $\bar{x}$, then $\partial_{P} f(\bar{x})$ is closed. The following theorem states an interesting property of a function $f$ which is p.l.n. at $\bar{x} \in \operatorname{dom}(f)$. Employing the following theorem, we can prove that if $f$ is p.l.n. at $\bar{x}$ and Lipschitz around $\bar{x}$, then it is regular at $\bar{x}$.

Theorem 3.5. Let $M$ be a Riemannian manifold, and $f: M \rightarrow(-\infty,+\infty]$ be p.l.n. at $\bar{x} \in \operatorname{dom}(f)$. Then for all $x$ in a neighborhood of $\bar{x}$,

$$
\partial_{L} f(x)=\partial_{P} f(x) .
$$

Proof. Since $f$ is p.l.n. at $\bar{x}$, we need only to prove the theorem at $\bar{x}$. Let $t_{0}>0$, $b>0$ and $\varepsilon>0$ be such that $B(\bar{x}, \varepsilon)$ is convex and

$$
f\left(x^{\prime}\right) \geq f(x)+\left\langle\exp _{x}^{-1}\left(x^{\prime}\right), v\right\rangle-t / 2 d\left(x^{\prime}, x\right)^{2}
$$

provided that $t>t_{0},\|v\|<b t, v \in \partial_{P} f(x), d\left(x^{\prime}, \bar{x}\right)<\varepsilon, d(x, \bar{x})<\varepsilon$. Suppose that $v \in \partial_{L} f(\bar{x})$. Then there exist sequences $\left\{x_{n}\right\}$ converging to $\bar{x}$, and $\left\{v_{n}\right\}$ converging to $v$ with $v_{n} \in \partial_{P} f\left(x_{n}\right)$. For all $i$ large enough, $\left\{\left\|L_{x_{i} \bar{x}}\left(v_{i}\right)\right\|\right\}$ is bounded. Therefore, one can deduce, for all $i$ large enough, that there is $t>t_{0}$ such that $\left\|v_{i}\right\| \leq b t$. Eventually, $d\left(x_{n}, \bar{x}\right)<\varepsilon$ and we pick $t>t_{0}$ such that $\left\|v_{n}\right\|<b t$. Thus,

$$
f(x) \geq f\left(x_{n}\right)+\left\langle\exp _{x_{n}}^{-1}(x), v_{n}\right\rangle-t / 2 d\left(x, x_{n}\right)^{2},
$$

for all $x$ with $d(x, \bar{x})<\varepsilon$. Taking the limit in (3.4) as $n \rightarrow \infty$, we get $v \in \partial_{P} f(\bar{x})$.

In Lemma 3.6 the relationship between prox-regular sets and p.l.n. functions on Riemannian manifolds is presented. The proof of it can be obtained along the same lines as [18, Proposition 2.1].

Lemma 3.6. A subset $S$ of a Riemannian manifold $M$ is prox-regular at $\bar{x} \in S$ if and only if the indicator function of $S$ is p.l.n. at $\bar{x}$. 
Now we are going to prove the regularity of prox-regular sets in Riemannian manifolds.

Lemma 3.7. Let $M$ be a Riemannian manifold and let $S \subset M$ be prox-regular at $\bar{x} \in S$. Then:

(a) $T_{S}^{B}(\bar{x}) \subseteq\left(N_{S}^{P}(\bar{x})\right)^{\circ}$.

(b) $N_{S}^{P}(\bar{x})=N_{S}^{L}(\bar{x})=N_{S}^{C}(\bar{x})$.

(c) $T_{S}^{B}(\bar{x})=T_{S}^{C}(\bar{x})$.

(d) $T_{S}^{B}(\bar{x})$ is a convex cone.

Proof. To prove (a), let $w=\lim \frac{z_{i}}{t_{i}}$ be in $T_{S}^{B}(\bar{x})$, and let $v \in N_{S}^{P}(\bar{x})$. Then there exist $\varepsilon>0$ and $\rho>0$ such that

$$
\left\langle\frac{\varepsilon v}{\|v\|}, \exp _{\bar{x}}^{-1}(y)\right\rangle \leq \rho / 2 d(y, \bar{x})^{2},
$$

for every $y$ in $B(\bar{x}, \varepsilon)$. For large enough $i, \exp _{\bar{x}}\left(z_{i}\right) \in B(\bar{x}, \varepsilon)$ and

$$
\left\langle\frac{\varepsilon v}{\|v\|}, \frac{z_{i}}{t_{i}}\right\rangle \leq \rho / 2 \frac{d\left(\exp _{\bar{x}}\left(z_{i}\right), \bar{x}\right)^{2}}{t_{i}} .
$$

By limiting, we deduce $\langle v, w\rangle \leq 0$, which completes the proof of (a).

Let us prove assertion (b). The set $S$ is prox-regular at $\bar{x}$; hence there exist $\varepsilon>0$ and $\rho>0$, such that $B(\bar{x}, \varepsilon)$ is convex and for every $x \in S \cap B(\bar{x}, \varepsilon)$ and $v \in N_{S}^{L}(x)$ with $\|v\|<\varepsilon$,

$$
\left\langle v, \exp _{x}^{-1}\left(x^{\prime}\right)\right\rangle \leq \rho / 2 d\left(x^{\prime}, x\right)^{2} \text {, for every } x^{\prime} \in S \cap B(\bar{x}, \varepsilon) .
$$

This can be deduced from Theorem 3.5. Lemma 3.6 and the convexity property of $N_{S}^{P}(\bar{x})$.

We turn to (c). It is easy to prove that $T_{S}^{C}(\bar{x})$ is a subset of $T_{S}^{B}(\bar{x})$. On the other hand, part (a) and part (b) imply $T_{S}^{B}(\bar{x}) \subseteq\left(N_{S}^{P}(\bar{x})\right)^{\circ}=\left(N_{S}^{L}(\bar{x})\right)^{\circ}=\left(N_{S}^{C}(\bar{x})\right)^{\circ}=$ $T_{S}^{C}(\bar{x})$, as required.

Assertion (d) can be obtained from part (c) and the convexity property of $T_{S}^{C}(\bar{x})$.

3.2. Hypomonotone mappings and their connections with prox-regular sets. In this subsection we recall the definitions of monotone and strongly monotone mappings on convex subsets of Riemannian manifolds; for more details see [3]. Then, we define hypomonotone mappings on convex subsets of Riemannian manifolds and find the relationship between monotone and hypomonotone mappings. Furthermore, prox-regular sets are characterized by the hypomonotone truncated limiting normal cone.

Definition 3.8. Let $M$ be a Riemannian manifold. A mapping $T: M \rightrightarrows T M^{*}$ with $T(x) \subset T_{x} M^{*}$ for every $x \in M$ is said to be monotone on a convex subset $U$ of $M$ if

$$
\left\langle v_{1}, \exp _{x_{1}}^{-1}\left(x_{2}\right)\right\rangle+\left\langle v_{2}, \exp _{x_{2}}^{-1}\left(x_{1}\right)\right\rangle \leq 0,
$$

provided that $x_{1}, x_{2} \in U, v_{1} \in T\left(x_{1}\right)$ and $v_{2} \in T\left(x_{2}\right)$. Moreover, $T$ is strongly monotone on $U$ if there exists $\sigma>0$ such that

$$
\left\langle v_{1}, \exp _{x_{1}}^{-1}\left(x_{2}\right)\right\rangle+\left\langle v_{2}, \exp _{x_{2}}^{-1}\left(x_{1}\right)\right\rangle \leq-\sigma d\left(x_{1}, x_{2}\right)^{2},
$$

provided that $x_{1}, x_{2} \in U, v_{1} \in T\left(x_{1}\right)$ and $v_{2} \in T\left(x_{2}\right)$. 
Definition 3.9. Let $M$ be a Riemannian manifold. A mapping $T: M \rightrightarrows T M^{*}$ with $T(x) \subset T_{x} M^{*}$ for every $x \in M$ is said to be hypomonotone on a convex subset $U$ of $M$ if there exists $\sigma>0$ such that

$$
\left\langle v_{1}, \exp _{x_{1}}^{-1}\left(x_{2}\right)\right\rangle+\left\langle v_{2}, \exp _{x_{2}}^{-1}\left(x_{1}\right)\right\rangle \leq+\sigma d\left(x_{1}, x_{2}\right)^{2},
$$

provided that $x_{1}, x_{2} \in U, v_{1} \in T\left(x_{1}\right)$ and $v_{2} \in T\left(x_{2}\right)$.

Similar definitions hold for a set-valued mapping $T$ defined from a Riemannian manifold $M$ to its tangent bundle $T M$.

Remark 2. Let $U$ be an open convex subset of a Riemannian manifold $M$ and let $y \in U$ be an arbitrary fixed point. The map $d^{2}(., y): U \rightarrow \mathbb{R}$ is $C^{1}$ and $\operatorname{grad}\left(\frac{1}{2} d^{2}(x, y)\right)=-\exp _{x}^{-1}(y)$, where grad denotes the gradient vector field. On the other hand, $-\exp _{x}^{-1}(y)=L_{y x}\left(\exp _{y}^{-1}(x)\right)$; see [1]. Moreover, $\operatorname{grad}\left(\frac{1}{2} d^{2}(x, y)\right)$ is strongly monotone with $\sigma=1$; see [7]. Hence

$$
\left\langle-\exp _{x_{1}}^{-1}(y), \exp _{x_{1}}^{-1}\left(x_{2}\right)\right\rangle+\left\langle-\exp _{x_{2}}^{-1}(y), \exp _{x_{2}}^{-1}\left(x_{1}\right)\right\rangle \leq-d\left(x_{1}, x_{2}\right)^{2} .
$$

Therefore, if $T: M \rightrightarrows T M$ with $T(x) \subset T_{x} M$ for every $x \in M$ is $\sigma$-hypomonotone on a convex subset $U$ and $y \in U$, then

$\left\langle v_{1}, \exp _{x_{1}}^{-1}\left(x_{2}\right)\right\rangle+\left\langle v_{2}, \exp _{x_{2}}^{-1}\left(x_{1}\right)\right\rangle \leq \sigma\left\langle\exp _{x_{1}}^{-1}(y), \exp _{x_{1}}^{-1}\left(x_{2}\right)\right\rangle+\left\langle\exp _{x_{2}}^{-1}(y), \exp _{x_{2}}^{-1}\left(x_{1}\right)\right\rangle$, which means $x \mapsto T(x)+\sigma L_{y x}\left(\exp _{y}^{-1}(x)\right)$ is monotone on $U$.

The next result provides a characterization for convexity in the case of a differentiable function.

Lemma 3.10. Let $U$ be a convex open subset of a Riemannian manifold $M$, and let $f: M \rightarrow \mathbb{R}$ be a differentiable function on $U$. Then $f$ is convex on $U$ if and only if the vector field $\operatorname{grad}(f)$ is monotone on $U$.

Proof. See [7].

Let us define for $r>0$ the mapping $N_{S}^{L^{r}}: M \rightrightarrows T M^{*}$ by $N_{S}^{L^{r}}(x)=N_{S}^{L}(x) \cap$ $B\left(0_{x}, r\right)$ if $x \in S$ and $N_{S}^{L^{r}}(x)=\emptyset$ if $x \notin S$. Note that $B\left(0_{x}, r\right)$ is the open ball in $T_{x} M^{*}$ of center $0_{x}$ and radius $r$.

The connection between a prox-regular subset $S$ of a Riemannian manifold $M$ and the mapping $N_{S}^{L^{r}}$ will now be established. The proof of it can be obtained along the same lines as [18, Theorem $1.3(\mathrm{~g})]$,

Theorem 3.11. A closed subset $S$ of a Riemannian manifold $M$ is prox-regular at $\bar{x} \in S$ if and only if there exist a real number $r>0$ and a convex neighborhood $U$ of $\bar{x}$ such that $N_{S}^{L^{r}}$ is hypomonotone on $U$.

3.3. Metric projection and distance function corresponding to the proxregular sets. Let $M$ be a Riemannian manifold and $S \subset M$ be a nonempty set. Let

$$
P_{S}(q)=\left\{p \in S: d(q, p)=d_{S}(p)\right\}
$$

be the set of metric projection of the point $q \in M$ to the set $S$. In this subsection we establish our main results. We prove the existence and properties of metric projection of prox-regular sets in Riemannian manifolds. Due to the theorem of Hopf-Rinow, if $M$ is a complete finite dimensional manifold, then any closed set $S \subset M$ is proximinal, i.e., $P_{S}(q) \neq \emptyset$ for all $q \in M$; see [14, p. 108]. 
First we prove that for every prox-regular set in a Riemannian manifold $M$, there exists an open subset containing $S$ on which the metric projection exists and is continuous.

Theorem 3.12. Let $S$ be a prox-regular subset of a Riemannian manifold $M$. Then there exists an open set $W$ containing $S$ such that $P_{S}$ is a single-valued continuous mapping on $W$.

Proof. For any $q \in M$, one can find the convex neighborhood $B\left(q, r_{q}\right)$ with compact closure such that $d(., q)^{2}$ is $C^{\infty}$ and strongly convex on $B\left(q, r_{q}\right)$; see [12, 23. On the other hand, according to Theorem 3.11, for every $q \in S$ there exist a real number $r_{q}^{\prime}>0$ and a convex neighborhood $U_{q}$ of $q$ such that $N_{S}^{L}{ }^{r_{q}^{\prime}}$ is hypomonotone on $U_{q}$. Hence, there exist $\rho_{q}>0$ and $\varepsilon_{q}$ with $0<\varepsilon_{q}<\min \left\{r_{q}, r_{q}^{\prime}\right\}$ such that

$$
\left\langle v_{1}, \exp _{p_{1}}^{-1}\left(p_{2}\right)\right\rangle+\left\langle v_{2}, \exp _{p_{2}}^{-1}\left(p_{1}\right)\right\rangle \leq \rho_{q} d\left(p_{1}, p_{2}\right)^{2},
$$

provided that $p_{i} \in B\left(q, \varepsilon_{q}\right), v_{i} \in N_{S}^{L}\left(p_{i}\right)$ and $\left\|v_{i}\right\|<\varepsilon_{q}, i=1,2$. Let $\lambda_{q}<$ $\min \left\{1, \rho_{q}\right\}$, and set

$$
W:=\bigcup_{q \in S} B\left(q, \lambda_{q} \varepsilon_{q} /\left(2 \rho_{q}\right)\right)
$$

The set $S$ is contained in the open set $W$, and for every $p \in W$ there exists $q \in S$ such that $p \in B\left(q, \lambda_{q} \varepsilon_{q} /\left(2 \rho_{q}\right)\right)$ and $d_{S}(p) \leq d(p, q)<\lambda_{q} \varepsilon_{q} /\left(2 \rho_{q}\right)$. Moreover, $\lambda_{q} \varepsilon_{q} /\left(2 \rho_{q}\right) \leq \varepsilon_{q} \leq r_{q}$, which implies that $B\left(q, \lambda_{q} \varepsilon_{q} /\left(2 \rho_{q}\right)\right) \subset B\left(q, r_{q}\right)$. Since $S$ is closed and the closure of $B\left(q, r_{q}\right)$ is compact, there exists a point $p^{\prime} \in S \cap$ $\overline{B\left(q, \lambda_{q} \varepsilon_{q} /\left(2 \rho_{q}\right)\right)} \subset S \cap \overline{B\left(q, r_{q}\right)}$ such that $d_{S}(p)=d\left(p, p^{\prime}\right)$. In particular $d_{S}(p) \leq$ $d(p, q)<\lambda_{q} \varepsilon_{q} /\left(2 \rho_{q}\right)$. Hence there exists $p^{\prime} \in S \cap B\left(q, \lambda_{q} \varepsilon_{q} /\left(2 \rho_{q}\right)\right)$ such that $p^{\prime} \in$ $P_{S}(p)$.

It remains to show that $P_{S}(p)=\left\{p^{\prime}\right\}$. We claim that

$$
\left\{\lambda \exp _{p^{\prime}}^{-1}(p): \lambda \geq 0\right\} \subseteq N_{S}^{P}\left(p^{\prime}\right) .
$$

Since $p^{\prime} \in P_{S}(p)$, it follows that for each $s \in S, d\left(p^{\prime}, p\right)^{2} \leq d(p, s)^{2}$. Moreover, let $\gamma$ be the unique geodesic connecting $p$ and $p^{\prime}$. Now for $q$ on $\gamma$ close enough to $p$, we define $\psi_{q}: M \rightarrow \mathbb{R}$ by

$$
\psi_{q}(s)=(d(q, s)+d(q, p))^{2},
$$

which is $C^{2}$ at $p^{\prime}$. For $s$ near $p^{\prime}$, let $\eta(t)=\exp _{p^{\prime}}(t v)$ be the unique geodesic connecting $p^{\prime}$ and $s$. Using Taylor's Theorem, there exists $q^{\prime}=\exp _{p^{\prime}}\left(t_{0} v\right)$ such that

$$
\begin{aligned}
0 \leq & d(p, s)^{2}-d\left(p^{\prime}, p\right)^{2} \leq \psi_{q}(s)-\psi_{q}\left(p^{\prime}\right) \\
\leq & \left\langle D \psi_{q}\left(p^{\prime}\right), \exp _{p^{\prime}}^{-1}(s)\right\rangle \\
& +\frac{1}{2}\left\langle D^{2} \psi_{q}\left(q^{\prime}\right) D \exp _{p^{\prime}}\left(t_{0} v\right)\left(\exp _{p^{\prime}}^{-1}(s)\right), D \exp _{p^{\prime}}\left(t_{0} v\right)\left(\exp _{p^{\prime}}^{-1}(s)\right)\right\rangle \\
\leq & \left\langle D \psi_{q}\left(p^{\prime}\right), \exp _{p^{\prime}}^{-1}(s)\right\rangle+\frac{1}{2} C_{q, q^{\prime}} d\left(p^{\prime}, s\right)^{2} \\
= & \left\langle 2 d\left(p, p^{\prime}\right) \frac{\partial d}{\partial y}\left(q, p^{\prime}\right), \exp _{p^{\prime}}^{-1}(s)\right\rangle+\frac{1}{2} C_{q, q^{\prime}} d\left(p^{\prime}, s\right)^{2},
\end{aligned}
$$


where $C_{q, q^{\prime}}$ is obtained as follows:

$$
D^{2} \psi_{q}\left(q^{\prime}\right)=2\left(d\left(q, q^{\prime}\right)+d(q, p)\right) \frac{\partial^{2} d}{\partial y^{2}}\left(q, q^{\prime}\right)+2\left(\frac{\partial d}{\partial y}\left(q, q^{\prime}\right)\right)^{2}
$$

and

Hence

$$
\left\|\frac{\partial^{2} d}{\partial y^{2}}\left(q, q^{\prime}\right)\right\|=\frac{1}{d\left(q, q^{\prime}\right)},\left\|\frac{\partial d}{\partial y}\left(q, q^{\prime}\right)\right\|=1 .
$$

$$
\left\|D^{2} \psi_{q}\left(q^{\prime}\right)\right\| \leq 2 \frac{d\left(q, q^{\prime}\right)+d(q, p)}{d\left(q, q^{\prime}\right)}+2=C_{q, q^{\prime}} .
$$

Also, by Gauss's lemma [13, Proposition 3.2],

$$
\left\langle D \exp _{p^{\prime}}\left(t_{0} v\right)\left(\exp _{p^{\prime}}^{-1}(s)\right), D \exp _{p^{\prime}}\left(t_{0} v\right)\left(\exp _{p^{\prime}}^{-1}(s)\right)\right\rangle=\left\|\exp _{p^{\prime}}^{-1}(s)\right\|^{2}=d\left(p^{\prime}, s\right)^{2} .
$$

By letting $q$ go to $p,\left\langle 2 d\left(p, p^{\prime}\right) \frac{\partial d}{\partial y}\left(q, p^{\prime}\right), \exp _{p^{\prime}}^{-1}(s)\right\rangle+\frac{1}{2} C_{q, q^{\prime}} d\left(p^{\prime}, s\right)^{2}$ tends to

$$
\left\langle 2 d\left(p, p^{\prime}\right) \frac{\partial d}{\partial y}\left(p, p^{\prime}\right), \exp _{p^{\prime}}^{-1}(s)\right\rangle+2 d\left(p^{\prime}, s\right)^{2} .
$$

Therefore

$$
\left\langle\exp _{p^{\prime}}^{-1}(p), \exp _{p^{\prime}}^{-1}(s)\right\rangle \leq d\left(p^{\prime}, s\right)^{2}, \text { for } s \text { close enough to } p^{\prime},
$$

and the proof of the claim is complete.

We now show that $P_{S}(p)=\left\{p^{\prime}\right\}$. We proceed by contradiction: let $p^{\prime \prime}$ be any point of $S$ satisfying $d\left(p, p^{\prime \prime}\right)=d_{S}(p)$ which belongs to $B\left(q, \lambda_{q} \varepsilon_{q} /\left(2 \rho_{q}\right)\right)$ and $p^{\prime} \neq p^{\prime \prime}$. Since the function $d(., q)^{2}$ is $C^{\infty}$ and strongly convex on $B\left(q, \lambda_{q} \varepsilon_{q} /\left(2 \rho_{q}\right)\right)$, by (3.6), the previous claim and Theorem 3.5, we deduce that

$$
\begin{aligned}
-\rho_{q} d\left(p^{\prime \prime}, p^{\prime}\right)^{2} & \leq\left\langle-\frac{2 \rho_{q}}{\lambda_{q}} \exp _{p^{\prime}}^{-1}(p), \exp _{p^{\prime}}^{-1}\left(p^{\prime \prime}\right)\right\rangle+\left\langle-\frac{2 \rho_{q}}{\lambda_{q}} \exp _{p^{\prime \prime}}^{-1}(p), \exp _{p^{\prime \prime}}^{-1}\left(p^{\prime}\right)\right\rangle \\
& \leq-2 \frac{\rho_{q}}{\lambda_{q}} d\left(p^{\prime \prime}, p^{\prime}\right)^{2}
\end{aligned}
$$

which means $\lambda_{q} \geq 2$, a contradiction.

Now we prove that $P_{S}$ is continuous on $W$. Suppose the sequence $\left\{p_{i}\right\} \subset W$ converges to $p_{0} \in W$. One can find $B\left(q, \lambda_{q} \varepsilon_{q} /\left(2 \rho_{q}\right)\right)$ such that $p_{0} \in B\left(q, \lambda_{q} \varepsilon_{q} /\left(2 \rho_{q}\right)\right)$. Hence except for a finite number of $p_{i}$, the others are in $B\left(q, \lambda_{q} \varepsilon_{q} /\left(2 \rho_{q}\right)\right)$. Thus we suppose that $p_{i} \in B\left(q, \lambda_{q} \varepsilon_{q} /\left(2 \rho_{q}\right)\right)$ for all $i=1,2, \ldots$ Moreover,

$$
d\left(P_{S}\left(p_{i}\right), q\right) \leq d\left(p_{i}, q\right)+d\left(P_{S}\left(p_{i}\right), p_{i}\right) \leq 2 d\left(p_{i}, q\right) \leq 2 \lambda_{q} \varepsilon_{q} /\left(2 \rho_{q}\right) \leq r_{q} .
$$

Therefore $P_{S}\left(p_{i}\right) \in B\left(q, r_{q}\right) \cap S$, which has compact closure. Consequently, $P_{S}\left(p_{i}\right)$ has a convergent subsequence to some point $p^{\prime} \in \overline{B\left(q, r_{q}\right) \cap S}$. Now $d\left(P_{S}\left(p_{i}\right), p_{i}\right)=$ $d_{S}\left(p_{i}\right) \rightarrow d_{S}\left(p_{0}\right)=d\left(p^{\prime}, p_{0}\right)$, which implies that $P_{S}\left(p_{0}\right)=p^{\prime}=\lim _{i} P_{S}\left(p_{i}\right)$, and the proof is complete.

Recall that a complete simply connected Riemannian manifold of nonpositive curvature is called a Hadamard manifold; for more details see [13.

Theorem 3.13. Let $S$ be a prox-regular subset of a Hadamard manifold $M$. If $q$ is a point of the boundary $S$ in $M$, and if $\lambda_{q} \varepsilon_{q} /\left(2 \rho_{q}\right)$ is as in the proof of Theorem [3.12, then there is a positive constant $C$ such that for any two points $p_{1}, p_{2}$ in $B\left(q, \lambda_{q} \varepsilon_{q} /\left(2 \rho_{q}\right)\right) \backslash S$,

$$
d\left(P_{S}\left(p_{1}\right), P_{S}\left(p_{2}\right)\right) \leq C d\left(p_{1}, p_{2}\right) .
$$


Proof. Let $p_{1}, p_{2}$ be arbitrary points in $B\left(q, \lambda_{q} \varepsilon_{q} /\left(2 \rho_{q}\right)\right) \backslash S$. In order to prove the theorem, we shall use Shapiro's variational principle; see 20 . Consider the optimization problems

$$
\min _{z \in S \cap B\left(q, \lambda_{q} \varepsilon_{q} /\left(2 \rho_{q}\right)\right)} d\left(p_{1}, z\right)^{2}=\min _{v \in \exp _{q}^{-1}\left(S \cap B\left(q, \lambda_{q} \varepsilon_{q} /\left(2 \rho_{q}\right)\right)\right)} d\left(p_{1}, \exp _{q}(v)\right)^{2}
$$

and

$$
\min _{z \in S \cap B\left(q, \lambda_{q} \varepsilon_{q} /\left(2 \rho_{q}\right)\right)} d\left(p_{2}, z\right)^{2}=\min _{v \in \exp _{q}^{-1}\left(S \cap B\left(q, \lambda_{q} \varepsilon_{q} /\left(2 \rho_{q}\right)\right)\right)} d\left(p_{2}, \exp _{q}(v)\right)^{2} .
$$

Let $P_{S}\left(p_{i}\right)=p_{i}^{\prime}, i=1,2$. Since $M$ is Hadamard, then by [13, Theorem IX. 4.8], for every $z \in S \cap B\left(q, \lambda_{q} \varepsilon_{q} /\left(2 \rho_{q}\right)\right)$,

$$
d\left(p_{1}, z\right)^{2}-d\left(p_{1}, p_{1}^{\prime}\right)^{2} \geq-2\left\langle\exp _{p_{1}^{\prime}}^{-1}\left(p_{1}\right), \exp _{p_{1}^{\prime}}^{-1}(z)\right\rangle+d\left(p_{1}^{\prime}, z\right)^{2} .
$$

From the prox-regularity property of $S$,

$$
\left\langle\frac{2 \rho_{q}}{\lambda_{q}} \exp _{p_{1}^{\prime}}^{-1}\left(p_{1}\right), \exp _{p_{1}^{\prime}}^{-1}(z)\right\rangle \leq \frac{1}{2} \rho_{q} d\left(p_{1}^{\prime}, z\right)^{2} .
$$

Hence it follows from (3.9) that

$$
d\left(p_{1}, z\right)^{2}-d\left(p_{1}, p_{1}^{\prime}\right)^{2} \geq\left(-\lambda_{q}+1\right) d\left(p_{1}^{\prime}, z\right)^{2} .
$$

Without loss of generality we suppose that $\exp _{q}^{-1}$ is $C$-Lipschitz on $B\left(q, \lambda_{q} \varepsilon_{q} /\left(2 \rho_{q}\right)\right)$. Therefore, if $\exp _{q}\left(w_{i}\right)=p_{i}^{\prime}, i=1,2$ and $\exp _{q}(v)=z$, then

$$
d\left(p_{1}, z\right)^{2}-d\left(p_{1}, p_{1}^{\prime}\right)^{2} \geq\left(-\lambda_{q}+1\right) d\left(p_{1}^{\prime}, z\right)^{2} \geq \frac{1}{C^{2}}\left(1-\lambda_{q}\right) d\left(w_{1}, v\right)^{2} .
$$

By Shapiro's variational principle,

$$
d\left(p_{1}^{\prime}, p_{2}^{\prime}\right) \leq C d\left(w_{1}, w_{2}\right) \leq 2 \frac{C^{3}}{1-\lambda_{q}} d\left(p_{1}, p_{2}\right) .
$$

Theorem 3.14. Let $S$ be a prox-regular subset of a Hadamard manifold $M$. Then for every $\sigma>0$ and $q \in S$, there is a convex neighborhood $U_{\sigma}$ around $q$ on which the function $d_{S}^{2}()+.\sigma d(., q)^{2}$ is convex.

Proof. We claim that for arbitrary points $q_{1}$ and $q_{2}$ in $B\left(q, \lambda_{q} \varepsilon_{q} /\left(2 \rho_{q}\right)\right)$, we have that $\partial_{P} d_{S}^{2}\left(q_{i}\right) \neq \emptyset$ for $i=1,2$. Otherwise, there exist $q_{1}^{\prime}$ and $q_{2}^{\prime}$ in $B\left(q, \lambda_{q} \varepsilon_{q} /\left(2 \rho_{q}\right)\right)$ such that for $i=1,2, \partial_{P} d_{S}^{2}\left(q_{i}^{\prime}\right)=\emptyset$. By the density theorem [2, Theorem 3.2], there are points $p_{1}$ and $p_{2}$ in $B\left(q, \lambda_{q} \varepsilon_{q} /\left(2 \rho_{q}\right)\right)$ such that for $i=1,2, \partial_{P} d_{S}^{2}\left(p_{i}\right) \neq \emptyset$. Then [2, Theorem 3.3] implies that $d_{S}^{2}$ is differentiable at these points. Moreover, $P_{S}\left(p_{i}\right)=p_{i}^{\prime}, i=1,2$. By [13, Theorem IX. 4. 8], Theorem 3.13 and Remark 2 one can deduce

$$
\begin{aligned}
& \left(\frac{4 C^{3}}{1-\lambda_{q}}-2\right)\left(\left\langle\exp _{p_{1}}^{-1}(q), \exp _{p_{1}}^{-1}\left(p_{2}\right)\right\rangle+\left\langle\exp _{p_{2}}^{-1}(q), \exp _{p_{2}}^{-1}\left(p_{1}\right)\right\rangle\right) \\
& \quad \geq-2 d\left(p_{1}, p_{2}\right)^{2}+4 \frac{C^{3}}{1-\lambda_{q}} d\left(p_{1}, p_{2}\right)^{2} \\
& \quad \geq-2 d\left(p_{1}, p_{2}\right)^{2}+2 d\left(p_{1}, p_{2}\right) d\left(p_{1}^{\prime}, p_{2}^{\prime}\right) \\
& \quad \geq-d\left(p_{1}, p_{1}^{\prime}\right)^{2}-d\left(p_{2}, p_{2}^{\prime}\right)^{2}-d\left(p_{1}, p_{2}\right)^{2}-d\left(p_{1}, p_{2}\right)^{2}+d\left(p_{1}^{\prime}, p_{2}\right)^{2}+d\left(p_{2}^{\prime}, p_{1}\right)^{2} \\
& \quad \geq-2\left\langle\exp _{p_{1}}^{-1}\left(p_{1}^{\prime}\right), \exp _{p_{1}}^{-1}\left(p_{2}\right)\right\rangle-2\left\langle\exp _{p_{2}}^{-1}\left(p_{2}^{\prime}\right), \exp _{p_{2}}^{-1}\left(p_{1}\right)\right\rangle .
\end{aligned}
$$


On the basis of Lemma 3.10 we conclude that $d_{S}^{2}+\left(\frac{2 C^{3}}{1-\lambda_{q}}-1\right) d(., q)^{2}$ is convex on $B\left(q, \lambda_{q} \varepsilon_{q} /\left(2 \rho_{q}\right)\right)$. This shows our goal, and it also in turn implies that $\partial_{P} d_{S}^{2}$ is nonempty-valued on $B\left(q, \lambda_{q} \varepsilon_{q} /\left(2 \rho_{q}\right)\right)$, which proves our claim.

Now, we define the notion of locally Lipschitz vector fields on Riemannian manifolds.

Definition 3.15. Let $M$ be a Riemannian manifold. A mapping $X: M \rightarrow T M$ satisfying $X_{y} \in T_{y} M$ for all $y \in M$ is said to be a Lipschitz vector field of rank $k$ near a given point $x \in M$ if for some $\varepsilon>0$, we have

$$
\left\|L_{y z} X(y)-X(z)\right\| \leq k d(y, z) \quad \text { for all } \quad z, y \in B(x ; \varepsilon),
$$

where $B(x ; \varepsilon)$ is convex, and $L_{y z}$ is the parallel transport along the unique geodesic connecting $z$ and $y$.

Note that if we consider the Riemannian metrics on $M$ and $T M$, then the above definition is equivalent to the usual definition of locally Lipschitz functions on metric spaces; see [4, p. 241]. Any two Riemannian metrics, each of which is bounded locally by a constant multiple of the other, give equivalent concepts of Lipschitz continuity though not the same local Lipschitz constant.

Remark 3. By Theorem 3.14 we deduce that $d_{S}^{2}$ is differentiable on the neighborhood $B\left(q, \lambda_{q} \varepsilon_{q} /\left(2 \rho_{q}\right)\right)$ which is defined in the proof of Theorem 3.12. Also [2. Theorem 3.3] implies that for every $p_{1} \in B\left(q, \lambda_{q} \varepsilon_{q} /\left(2 \rho_{q}\right)\right)$ with $P_{S}\left(p_{1}\right)=p_{1}^{\prime}$, $\operatorname{grad}\left(d_{S}^{2}\right)\left(p_{1}\right)=2 d\left(p_{1}, p_{1}^{\prime}\right) \frac{\partial d}{\partial x}\left(p_{1}, q_{1}\right)$, where $q_{1}$ is on the unique geodesic connecting $p_{1}$ and $p_{1}^{\prime}$ and closed enough to $p_{1}$. Indeed, $X(p)=\frac{\partial d}{\partial x}\left(p, q_{p}\right)$ is the unit tangent to the unique minimizing geodesic segment from $p$ to $P_{S}(p)$, where $q_{p}$ is on the unique geodesic connecting $p$ and $P_{S}(p)$ and closed enough to $p$. Along the same lines as [8, Proposition 4.1] one can prove that the vector field $X$ is locally Lipschitz on $W \backslash S$, where $W$ and $S$ are defined as in the proof of Theorem 3.12

Theorem 3.16. Let $S$ be a prox-regular subset of a Hadamard manifold $M$. Then there exists an open set $W$ containing $S$ such that $d_{S}^{2}$ is $C^{1+}$ on $W \backslash S$; i.e., $d_{S}^{2}$ is differentiable on $W \backslash S$ with the locally Lipschitz gradient vector field $\operatorname{grad}\left(d_{S}^{2}\right)$ : $W \backslash S \rightarrow T M$.

Proof. Consider the set $B\left(q, \lambda_{q} \varepsilon_{q} /\left(2 \rho_{q}\right)\right)$ which is defined in the proof of Theorem 3.12. Let $p_{1}, p_{2}$ be arbitrary points of $B\left(q, \lambda_{q} \varepsilon_{q} /\left(2 \rho_{q}\right)\right)$. Assume that the vector field $X$ defined in Remark 3 is $k_{q}$ Lipschitz on $B\left(q, \lambda_{q} \varepsilon_{q} /\left(2 \rho_{q}\right)\right)$. Then without loss of generality we suppose that $d\left(p_{2}, p_{2}^{\prime}\right) \leq d\left(p_{1}, p_{1}^{\prime}\right)$. Thus

$$
\begin{aligned}
& \left\|L_{p_{1} p_{2}}\left(2 d\left(p_{1}, p_{1}^{\prime}\right) \frac{\partial d}{\partial x}\left(p_{1}, q_{1}\right)\right)-2 d\left(p_{2}, p_{2}^{\prime}\right) \frac{\partial d}{\partial x}\left(p_{2}, q_{2}\right)\right\| \\
& \quad \leq 2 d\left(p_{1}, p_{1}^{\prime}\right)\left\|L_{p_{1} p_{2}}\left(\frac{\partial d}{\partial x}\left(p_{1}, q_{1}\right)\right)-\frac{\partial d}{\partial x}\left(p_{2}, q_{2}\right)\right\|+2\left\|d\left(p_{1}, p_{1}^{\prime}\right)-d\left(p_{2}, p_{2}^{\prime}\right)\right\| \\
& \quad \leq 2 k_{q} d\left(p_{1}, p_{1}^{\prime}\right) d\left(p_{1}, p_{2}\right)+2\left(d\left(p_{1}, p_{2}^{\prime}\right)-d\left(p_{2}, p_{2}^{\prime}\right)\right) \\
& \quad \leq 2 k_{q} d\left(p_{1}, p_{1}^{\prime}\right) d\left(p_{1}, p_{2}\right)+2 d\left(p_{1}, p_{2}\right) \leq\left(\frac{\lambda_{q} \varepsilon_{q} k_{q}}{\rho_{q}}+2\right) d\left(p_{1}, p_{2}\right),
\end{aligned}
$$

which completes the proof. 


\section{REFERENCES}

[1] D. Azagra, J. Ferrera, F. López-Mesas, Nonsmooth analysis and Hamilton-Jacobi equations on Riemannian manifolds, J. Funct. Anal. 220 (2005) 304-361. MR2119282 (2005k:49045)

[2] D. Azagra, J. Ferrera, Proximal calculus on Riemannian manifolds, Mediterr. J. Math. 2 (2005) 437-450. MR2192524(2007a:49023)

[3] A. Barani, M. R. Pouryayevali, Invariant monotone vector fields on Riemannian manifolds, Nonlinear Anal. 70 (2009) 1850-1861. MR2492123 (2010e:46038)

[4] R. D. Canary, D. B. A. Epstein, A. Marden, Fundamentals of Hyperbolic Geometry: Selected Expositions, Cambridge University Press, 2006. MR2230672 (2007c:57002)

[5] F. H. Clarke, Yu. S. Ledayaev, R. J. Stern, P. R. Wolenski, Nonsmooth Analysis and Control Theory, Graduate Texts in Mathematics, Vol. 178, Springer, New York, 1998. MR.1488695 (99a:49001)

[6] M. P. do Carmo, Riemannian Geometry, Birkhäuser, Boston, 1992. MR1138207 (92i:53001)

[7] J. X. Da Cruz Neto, O. P. Ferreira, L. R. Lucambio Pérez, Contributions to the study of monotone vector fields, Acta Math. Hungar. 94 (2002) 307-320. MR.1905099 (2003e:58023)

[8] R. E. Greene, K. Shiohama, Convex functions on complete noncompact manifolds: topological structure, Invent. Math. 63 (1981) 129-157. MR608531(82e:53065)

[9] S. Grognet, Théorème de Motzkin en courbure négative, Geom. Dedicata 79 (2000) 219-227. MR.1748883 (2001a:53066)

[10] S. Hosseini, M. R. Pouryayevali, Generalized gradients and characterization of epi-Lipschitz sets in Riemannian manifolds, Nonlinear Anal. 74 (2011) 3884-3895. MR2802974

[11] W. Klingenberg, Riemannian Geometry, Walter de Gruyter Studies in Mathematics, Vol. 1, Walter de Gruyter, Berlin, New York, 1982. MR666697 (84j:53001)

[12] S. Kobayashi, K. Nomizu, Foundations of Differential Geometry, Vol. II, Wiley-Interscience, New York, 1969. MR0238225(38:6501)

[13] S. Lang, Fundamentals of Differential Geometry, Graduate Texts in Mathematics, Vol. 191, Springer, New York, 1999. MR 1666820 (99m:53001)

[14] J. M. Lee, Riemannian Manifolds, An Introduction to Curvature, Graduate Texts in Mathematics, Vol. 176, Springer, 1997. MR1468735(98d:53001)

[15] D. Motreanu, N. H. Pavel, Quasitangent vectors in flow-invariance and optimization problems on Banach manifolds. J. Math. Anal. Appl. 88 (1982) 116-132. MR661406 (83h:58086)

[16] S. Z. Németh, Monotone vector fields, Publ. Math. Debrecen 54 (1999) 437-449. MR1694468 (2000g:53041)

[17] R. A. Poliquin, R. T. Rockafellar, Prox-regular functions in variational analysis, Trans. Amer. Math. Soc. 348 (1996) 1805-1838. MR1333397 (96h:49039)

[18] R. A. Poliquin, R. T. Rockafellar, L. Thibault, Local differentiability of distance functions, Trans. Amer. Math. Soc. 352 (2000) 5231-5249. MR1694378 (2001b:49024)

[19] A. Shapiro, Existence and differentiability of metric projections in Hilbert spaces. SIAM J. Optim. 4 (1994) 130-141. MR.1260410 (94m:90111)

[20] A. Shapiro, Perturbation analysis of optimization problems in Banach spaces, Numer. Funct. Anal. Optim. 13 (1992) 97-116. MR1163320 (93c:49019)

[21] R. Walter, On the metric projection onto convex sets in Riemannian spaces, Arch. Math. (Basel) 25 (1974) 91-98. MR0397631 (53:1490)

[22] J. Wang, G. López, V. Martín-Márquez, C. Li, Monotone and accretive vector fields on Riemannian manifolds, J. Optim. Theory Appl. 146 (2010) 691-708. MR2720608 (2012b:47217)

[23] J. H. C. Whitehead, Convex regions in the geometry of paths. Q. J. Math. 3 (1932) 33-42. IRAN

Department of Mathematics, University of Isfahan, P. O. Box 81745-163, Isfahan,

E-mail address: somayeh-hosseini@hotmail.com IRAN

Department of Mathematics, University of Isfahan, P. O. Box 81745-163, Isfahan,

E-mail address: pourya@math.ui.ac.ir 